\title{
Construcción semántica del concepto retos para las ciudades y comunidades sustentables por estudiantado universitario
}

\author{
Semantic Construction of the Concept Challenges for Sustainable Cities and Communities \\ by University Students
}

\section{Construção semântica do concepto desafios para cidades e comunidades sustentáveis por estudantes universitários}

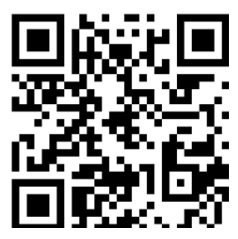

Pedro César Cantú-Martínez Universidad Autónoma de Nuevo León

Facultad de Ciencias Biológicas

Nuevo León, México

cantup@hotmail.com

https://orcid.org/0000-0001-8924-5343

Recibido • Received • Recebido: 28 / 12 / 2019

Corregido • Revised • Revisado: 25 / 07 / 2021

Aceptado • Accepted • Aprovado: 25 / 08 / 2021

\begin{abstract}
Resumen:
Introducción. Hoy en día, la problemática ambiental exige tomar acciones inmediatas a nuestra sociedad. Es así como se pronuncian los Objetivos del Desarrollo Sustentable en el año 2015, con el fin de orientar el desarrollo humano en el mundo, donde se privilegie la sustentabilidad. Objetivo: El propósito del presente artículo es mostrar la representación semántica de los desafíos que surgen, y así, cumplir con el objetivo 11 ciudades y comunidades sustentables, a través de la perspectiva de estudiantado universitario. Metodología: La indagación fue exploratoria y, para esto, se recurrió a la metodología de redes semánticas naturales. Se consideró a 59 jóvenes de universidad del área de ciencias biológicas de la Universidad Autónoma de Nuevo León. Resultados: La población fue de $66,1 \%$ mujeres y $33,9 \%$ hombres, y las edades fueron entre 18 y 28 años. Los principales retos expresados por los sujetos participantes correspondieron a la contaminación del aire, basura, contaminación del agua, deforestación, tráfico de autos, carencia de áreas verdes y sobrepoblación. Subsiste además una similitud del $75 \%$ entre las expresiones comentadas por las mujeres y hombres. Discusión: Las expresiones verbales aludidas por el estudiantado universitario determinan la presencia de una memoria semántica, la cual recrea la conjunción de sus experiencias, ideas y percepciones en relación con su entorno social y ambiental. Conclusiones: Se concluye que el constructo edificado por el estudiantado concuerda con las metas del objetivo 11 suscritas en la Agenda 2030.
\end{abstract}

Palabras claves: Construcción semántica; desafíos; desarrollo sustentable; comunidades; ciudades; estudiantado universitario. 
http://doi.org/10.15359/ree.25-3.37

http://www.una.ac.cr/educare

educare@una.ac.cr

\begin{abstract}
:
Introduction. Today, the environmental problem requires our society to take immediate action. Thus, the Sustainable Development Goals were pronounced in 2015 to guide human development in the world, where sustainability is privileged. Objective. The purpose of this article is to show the semantic representation of the challenges that arise to meet the goal of 11 sustainable cities and communities through the perspective of college students. Method. The inquiry was exploratory; this was done using the methodology of natural semantic networks and including 59 young college students from the life sciences area of the Autonomous University of Nuevo León. Results. The population was $66.1 \%$ women and $33.9 \%$ men, and the ages were between 18 and 28 years old. The main challenges expressed by the participants were air pollution, litter, water pollution, deforestation, car trafficking, lack of green areas, and overpopulation. There was also a 75\% similarity between the expressions commented by women and men. Discussion. Verbal expressions referred to by university students determine the presence of a semantic memory that recreates the conjunction of their experiences, ideas, and perceptions in relation to their environment. Conclusions. It is concluded that the construct edified by the students is consistent with the goals of goal 11 subscribed to the 2030 Agenda.
\end{abstract}

Keywords: Semantic construction; challenges; sustainable development; communities; cities; university students.

\title{
Resumo:
}

Introdução. Atualmente, os problemas ambientais exigem que nossa sociedade tome medidas imediatas. Assim, encontramos nos Objetivos de Desenvolvimento Sustentável de 2015, orientar o desenvolvimento humano no mundo, onde a sustentabilidade é privilegiada. Objetivo. $\mathrm{O}$ objetivo deste artigo é mostrar a representação semântica dos desafios que surgem para atingir a meta de 11 cidades e comunidades sustentáveis através da perspectiva dos estudantes universitários. Metodologia. O questionário foi exploratório, utilizando a metodologia das redes semânticas naturais onde foram consideradas 59 pessoas universitários da área de ciências da vida da Universidade Autônoma de Nuevo León. Resultados. A população foi de $66,1 \%$ de mulheres e 33,9\% de homens, com idades entre 18 e 28 anos. Os principais desafios expressos pelas pessoas participantes foram poluição do ar, lixo, poluição da água, desmatamento, tráfego de automóveis, falta de áreas verdes e superpopulação. Há também uma semelhança de $75 \%$ entre as expressões comentadas por mulheres e homens. Discussão. Expressões verbais referidas por estudantes universitários determinam a presença de uma memória semântica, que recria a conjunção de suas experiências, ideias e percepções em relação ao seu ambiente socioambiental. Conclusão. Conclui-se que a construção edificada pelos estudantes é coerente com as metas do objetivo 11 inscritos na Agenda 2030.

Palavras-chave: Construção semântica; desafios; desenvolvimento sustentável; comunidades; cidades; estudantes universitários.

\section{Introducción}

En el contexto internacional actual, desde distintos sectores que constituyen la sociedad se ha prodigado -esencialmente desde 1972 en el siglo XX- la imperiosa necesidad de plantear un nuevo tipo de desarrollo, el cual permita favorecer la perpetuidad del ser humano y de todo 
aquello que le rodea para su subsistencia. Este aspecto fue acogido y cristalizado en la Reunión de Río 92. Así pues, se nombró como desarrollo sustentable, el cual tiene como propósito "asegurar que satisfaga las necesidades del presente sin comprometer la capacidad de las futuras generaciones" (Naciones Unidas. Asamblea General, 1987, p. 23).

En este sentido, Aznar Minguet et al. (2014, citando a Borne, 2010), comentan que, para considerar una sociedad sustentable, esta debe:

conservar los sistemas que hacen posible la vida y la biodiversidad, asegurar que el uso de los recursos renovables sea menor que la capacidad del medio ambiente para renovarlos, minimizar la destrucción de los recursos no renovables y disponer de tecnología para sustituirlos, mantener la capacidad de transporte de los ecosistemas y, propiciar un equilibrio económico capaz de asegurar la justicia social. (p. 14)

De esta manera, la sociedad humana debe de conocer los preceptos que deben demarcar este nuevo derrotero, así como la formación de un nuevo capital humano que permita revertir la "crisis resultado del diálogo entre individuos y medio; entre cultural y natural. Un panorama donde se hacen evidentes asimetrías globales que tienen como resultado conflictos sociales, movimientos migratorios, deterioro de ecosistemas" (Bonil et al., 2010, p. 199), entre otros aspectos preponderantes, como hace mención Bonil et al. (2010).

Por esta razón, durante el 2015 se erigieron los Objetivos del Desarrollo Sustentable, (ODS), con la finalidad de "poner fin a la pobreza, proteger el planeta y garantizar que todas las personas gocen de paz y prosperidad para 2030" como lo comenta el Programa de las Naciones Unidas para el Desarrollo (Programa de las Naciones Unidas para el Desarrollo [PNUD], 2019a, párr. 1). Estos 17 ODS dan cuenta metas y acciones a emprender por nuestra sociedad donde se privilegie el equilibrio sustentable entre la dimensión social, la económica y la ambiental.

Por lo tanto, definitivamente se torna relevante examinar en estudiantes universitarios como se vinculan estos y se relacionan con su entorno socioambiental, el cual da cuenta también mediante expresiones verbales representativas de la conexión con este ambiente construido o por edificar. De esta manera, en el presente trabajo se pretende analizar la estructuración de los retos que los participantes expresan para el logro de las ciudades y comunidades sustentables, concepto que se establece como guía en el ODS número 11. Para alcanzar este propósito, se emplea la metodología de las redes semánticas naturales.

\section{Marco conceptual}

La ciudad, desde la perspectiva de Bottino Bernardi (2009), se constituye en un espacio territorial sumamente complejo, delimitada por una disposición administrativa o un decreto de orden político, donde se suceden cambios demográficos, económicos, sociales y culturales. 
http://doi.org/10.15359/ree.25-3.37

http://www.una.ac.cr/educare

educare@una.ac.cr

Además, ostenta, por y con sus integrantes, una honda exigencia de transformación y resignificación del contexto territorial, con un estilo de vida -preferentemente comercial e industrial- que, finalmente, conlleva una especialización del trabajo.

Hoy día, por el Programa de las Naciones Unidas para el Desarrollo (PNUD, 2019b) se advierte que más de la mitad de la población en el mundo cohabita en las ciudades, y cuya cifra ronda los 4.5 mil millones de personas. Por consiguiente, las ciudades también se alzan con el 80\% del consumo de la producción de energía que se genera en el mundo. Y, por consecuencia, también se constituyen en áreas emisoras de carbono, atribuyéndoseles, por lo menos, el 70\% de las emisiones que subsisten en el planeta. Al mismo tiempo, las ciudades se constituyen en sitios donde subsisten cerca de 828 millones de personas en condiciones de marginación y paupérrimamente.

En este sentido, es así como las ciudades se han constituido en espacios físicos que continúan extendiéndose, lo cual conlleva impactos ambientales bastante fehacientes al entorno natural. Estas manifestaciones están relacionadas a zonas urbanas totalmente deterioradas, como también a problemáticas relativas a la movilidad urbana, inundaciones, calidad del aire, violencia y discriminación social.

Ahora bien, bajo la perspectiva Simmel (citado por Charry Joya, 2006, p. 212), se puede mencionar otro rasgo que se ha exacerbado el contexto del individualismo y la ciudad, que ha llevado a grados superlativos la competencia, es decir, al punto que "la vida de la ciudad ha transformado la lucha con la naturaleza para la adquisición de alimento en una lucha [franca entre los propios seres humanos]". Por ello, en las últimas décadas, se ha tornado importante comprender las relaciones que subsisten entre las personas, sus actividades y el entorno natural. Sin embargo, se rescata, esencialmente, cómo estas construyen su realidad, la cual consta de amalgamar las percepciones, ideas y experiencias.

De esta forma, el llevar a cabo una aproximación de la memoria lexicográfica como también de sus conceptualizaciones esgrimidas mediante palabras, según Rodríguez (2010), se sostiene la contextualización de una realidad por el colectivo o grupo humano que lo expresa. Por lo tanto, Cantú-Martínez (2019), señala que estos enunciados son expresiones que representan también la construcción social e histórica de las personas de acuerdo con el contexto en que coexisten, dando certeza y representación -mediante el lenguaje- de lo que sucede en su derredor.

Por esta razón, las redes semánticas responden a un artilugio metodológico adecuado para acercarnos al significado colectivo, siempre relacionado a la edificación de una situación particular a partir de un grupo de personas. Aquí, resulta notorio el espacio funcional en que se subsiste $y$, también, expone el espacio de conciencia, el cual influye a la manera que la gente aprecia su entorno. 
http://doi.org/10.15359/ree.25-3.37

\section{Metodología}

La presente indagación fue de representación exploratoria, con el propósito de averiguar y consignar la percepción que exteriorizan 59 estudiantes universitarios (tanto mujeres como hombres) de la Facultad de Ciencias Biológicas de la Universidad Autónoma de Nuevo León (Nuevo León, México), en correspondencia con la noción retos de las ciudades y comunidades sustentables. La orientación de esta investigación fue de carácter bimodal, lo cual incumbe tanto una exploración cualitativa como cuantitativa.

Para llevar a cabo este estudio, se recurrió a la metodología de las redes semánticas naturales (Vera-Noriega et al., 2005). Esta metodología consiste en utilizar una expresión detonante -en este caso, retos de las ciudades y comunidades sustentables- para que los participantes determinen cuales serían, en cinco palabras. Más adelante, tras previa autorización verbal de este grupo, se aplicó un cuestionario donde se recogió información sociodemográfica, así como también las cinco palabras que se constituirían en los retos de las ciudades y comunidades sustentables. Posteriormente, se pidió que ponderaran de mayor a menor importancia. La de mayor importancia tendrá un valor de 5, y así hasta llegar a la de menor relevancia con un valor de 1.

En seguida, se llevó a cabo el análisis de la información recabada con el propósito de obtener el valor J que representa la riqueza semántica, el valor M que detenta el peso semántico de cada una de las palabras inscritas como vocablos definidores, y el Grupo SAM que concierne al grupo de palabras con mayores pesos específicos. Aquí se consideraron solo aquellas que

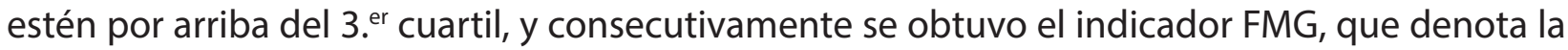
distancia en porcentaje existente entre las palabras (Cantú-Martínez, 2019, p. 4). Esto permitió conjuntar, a partir de la opinión de los estudiantes universitarios, el grupo de enunciados relacionados con el concepto detonante.

Se realizó una correlación de Spearman para corroborar si existiese asociación entre el número de menciones y el peso semántico de las palabras que se agrupan en el Grupo SAM. Finalmente, se evaluó la similaridad de las menciones hechas entre los hombres y las mujeres mediante el coeficiente de Jaccard (Cantú-Martínez, 2019), para, finalmente, llevar a cabo una matriz de entre los términos del Grupo SAM, tanto poblacional como por género, con las metas del Objetivo 11 de los ODS.

\section{Resultados}

\section{Población en general}

El número de participantes estuvo constituido por 59 estudiantes universitarios del área de ciencias biológicas, lo cual obedeció a una muestra intencional que consistió del $66.1 \%$ ( $f=39)$ de mujeres y $33.9 \%(f=20)$ de varones. La edad promedio de los y las partícipes en el 
http://doi.org/10.15359/ree.25-3.37

http://www.una.ac.cr/educare

educare@una.ac.cr

estudio tuvieron una edad promedio de $20( \pm 3)$ años, cuyas edades fluctuaron entre 18 y 28 años. Este grupo participante cursaba el tercer semestre de la carrera de químico bacteriólogo parasitólogo. Tras haber realizado la normalización -la cual consiste en revisar las expresiones y conjuntar aquellas que refieren a un mismo enunciado-, se halló un valor $J$ de 28 expresiones que fueron referidas al pensamiento detonante retos de las ciudades y comunidades sustentables.

Al indagar, se advirtió que las primeras cinco representaciones con mayor peso semántico, de mayor a menor, correspondieron a contaminación del aire cuyo peso fue de 123, basura con un peso de 109, contaminación del agua que tuvo un peso de 70, deforestación con un peso referenciado de 67 y finalmente el tráfico de autos con un peso 49; como se puede notar en la Tabla 1.

Mientras, el Grupo SAM, el cual agrupa las expresiones que los participantes consideraron como los retos más relevantes que enfrentarán las ciudades y las comunidades sustentables, incluyó la contaminación del aire, basura, contaminación del agua, deforestación, tráfico de autos, carencia de áreas verdes y, por último, la sobrepoblación. La noción definidora con mayor valor FMG porcentual fue contaminación del aire con un peso de 123 dentro del Grupo SAM. Además, fue mencionada por el $57.6 \%$ de los y las estudiantes participantes, como se observa en la Tabla 1.

Tabla 1: Grupo SAM, valor J, menciones, valor M e indicador FMG del constructo retos de las Ciudades y Comunidades Sustentables, señalado por la población en general

\begin{tabular}{lccc}
\multicolumn{1}{c}{ Grupo SAM } & Menciones & Valor M & Indicador FMG \\
\hline Contaminación del aire & 34 & 123 & 100 \\
Basura & 32 & 109 & 88.6 \\
Contaminación del agua & 23 & 70 & 56.9 \\
Deforestación & 22 & 67 & 54.5 \\
Tráfico de autos & 25 & 49 & 39.8 \\
Carencia de áreas verdes & 14 & 45 & 36.6 \\
Sobrepoblación & 12 & 36 & 29.3 \\
Valor J = 28 & & 3er Cuartil= 35 & \\
\hline
\end{tabular}

Nota: Elaboración propia. 
http://doi.org/10.15359/ree.25-3.37

En lo que refiere a la correlación entre las menciones expresadas y el valor $M$, se pudo constatar que estas se comportan en el mismo orden. En otras palabras, a mayor mención expresada mayor es su valor M, lo cual queda comprobado mediante el valor de correlación de Sperman de 0.89 ( $p<0.05$ ). Asimismo, se constató que la todas las expresiones vertidas por los participantes referentes a los retos más notables que afrontarán las ciudades y las comunidades, correspondieron -convenientemente- a las metas expresadas en el ODS 11 como se puede ver en la Tabla 2.

Tabla 2: Grupo SAM del constructo retos de las Ciudades y Comunidades Sustentables, señalado por la población en general y su congruencia con las metas del ODS 11

\begin{tabular}{|c|c|c|}
\hline Grupo SAM & Meta del ODS 11 & Descripción* \\
\hline Contaminación del aire & 11.6 & $\begin{array}{l}\text { Reducir el impacto ambiental negativo per capita en las ciudades, } \\
\text { atención especial a la calidad del aire y gestión de desechos } \\
\text { municipales y de otro tipo. }\end{array}$ \\
\hline Basura & 11.6 & $\begin{array}{l}\text { Reducir el impacto ambiental negativo per capita en las ciudades, } \\
\text { atención especial a la calidad del aire y gestión de desechos } \\
\text { municipales y de otro tipo. }\end{array}$ \\
\hline Contaminación del agua & 11.6 & Reducir el impacto ambiental negativo en las ciudades \\
\hline Deforestación & 11.4 & Redoblar esfuerzos para salvaguardar el patrimonio natural \\
\hline Tráfico de autos & 11.2 & $\begin{array}{l}\text { Proporcionar acceso a sistemas de transportes sustentables de } \\
\text { orden público. }\end{array}$ \\
\hline Carencia de áreas verdes & 11.7 & $\begin{array}{l}\text { Proporcionar acceso universal a zonas y espacios públicos. } \\
\text { Verdes. }\end{array}$ \\
\hline Sobrepoblación & 11.3 & Planificar y generar asentamientos humanos sostenibles \\
\hline
\end{tabular}

Nota: Elaboración propia con información de PNUD (2019b).

\section{Por género}

Enel grupo de mujeres, se encontró, posterior a la normalización, un valor de J de 28 vocablos relativos al concepto de retos de las Ciudades y Comunidades Sustentables. Adicionalmente, se encontró que las primeras cinco palabras expresadas con mayor peso semántico yacen en las expresiones, de mayor a menor, en contaminación del aire con un peso de 87, basura con un peso de 79 y, posteriormente, deforestación con un peso de 37, continuando el tráfico de autos y contaminación del agua con un peso de 34 cada una, como se aprecia en la Tabla 3. 
http://doi.org/10.15359/ree.25-3.37

http://www.una.ac.cr/educare

educare@una.ac.cr

En lo que respecta al Grupo SAM, que alberga los aspectos más relevantes a considerar en el marco de la concepción de los retos de las Ciudades y Comunidades Sustentables, se tomó en cuenta la contaminación del aire, basura, deforestación, el tráfico de autos, contaminación del agua, la carencia de áreas verdes y finalmente la sobrepoblación, como se puede observar en la Tabla 3. La palabra definidora con mayor valor FMG recayó en la contaminación del aire que ostentó un peso de 87 dentro del Grupo SAM y que fue aludida por el $64.1 \%$ de las mujeres.

Tabla 3: Grupo SAM, valor J, menciones, valor M e indicador FMG del constructo retos de las Ciudades y Comunidades Sustentables, señalado por las mujeres

\begin{tabular}{lccc}
\multicolumn{1}{c}{ Grupo SAM } & Menciones & Valor M & Indicador FMG \\
\hline Contaminación del aire & 25 & 87 & 100 \\
Basura & 21 & 79 & 90.8 \\
Deforestación & 13 & 37 & 42.5 \\
Tráfico de autos & 14 & 34 & 39.0 \\
Contaminación del agua & 14 & 34 & 39.0 \\
Carencia de áreas verdes & 9 & 29 & 33.3 \\
Sobrepoblación & 9 & 29 & 33.3 \\
Valor J= 28 & & 3er Cuartil= 28.5 & \\
\hline
\end{tabular}

Nota: Elaboración propia.

Entre tanto, los varones, tras conmutar y realizar la normalización, arrojaron un valor de $\mathrm{J}$ de 28 expresiones mancomunadas al concepto de los retos de las Ciudades y Comunidades Sustentables. También, se halló que las primeras cinco nociones manifestadas con mayor peso semántico recaen, de mayor a menor, en contaminación del aire y contaminación del agua (ambas con un peso de 36), continuando la basura y la deforestación con un peso cada una de 30, luego la carencia de áreas verdes con un peso de 16, como se puede advertir en la Tabla 4.

Por otro lado, en lo concerniente al Grupo SAM, lo varones consideraron como los principales retos de las Ciudades y Comunidades Sustentables, la contaminación del aire, contaminación del agua, basura, deforestación, tráfico de autos y la carencia de infraestructura vial, como puede ver en la Tabla 4. El vocablo definidor fue el que tuvo mayor valor FMG, en este caso, contaminación del aire y contaminación del agua, las cuales concurrieron en el $45 \%$ de las menciones de los varones. 
http://doi.org/10.15359/ree.25-3.37

Tabla 4: Grupo SAM, valor J, menciones, valor M e indicador FMG del constructo retos de las Ciudades y Comunidades Sustentables, señalado por los hombres

\begin{tabular}{lccc}
\hline Grupo SAM & Menciones & Valor M & Indicador FMG \\
\hline Contaminación del aire & 9 & 36 & 100 \\
Contaminación del agua & 9 & 36 & 100 \\
Basura & 11 & 30 & 83.3 \\
Deforestación & 9 & 30 & 83.3 \\
Carencia de áreas verdes & 5 & 16 & 44.4 \\
Tráfico de autos & 11 & 15 & 41.7 \\
Falta de Infraestructura vial & 9 & 14 & 38.9 \\
Valor J = 28 & & 3er Cuartil= 13.2 & \\
\hline
\end{tabular}

Nota: Elaboración propia.

En lo que se relaciona al nivel de similaridad entre el conjunto de expresiones del Grupo SAM de las mujeres y de los varones, se obtuvo un coeficiente de Jaccard de 0.75 , el cual implica que subsiste una similitud del 75\% entre las expresiones suscritas por las mujeres con respecto a la de los varones. Por otra parte, la similaridad entre las opiniones vertidas en el Grupo SAM de la población en General con respecto a las mujeres y varones, correspondieron valores del 100\% para las mujeres y de solo un $75 \%$ con respecto a los varones.

\section{Discusión}

Sin lugar a dudas, el modelo de desarrollo imperante favoreció el arraigo de hábitos y aspiraciones sociales que no coadyuvan al bienestar y, en cambio, colocan en riesgo la calidad ambiental y la progresión de una sustentabilidad en el tiempo. Esto conlleva a un deterioro de las dimensiones de carácter social, económico y ecológico, lo cual es evidente en las ciudades. Por este motivo, se ha erigido el Objetivo 11 de los ODS, el cual tiene como fin "mantener ciudades de manera que sigan creando empleos y prosperidad sin ejercer presión sobre la tierra y los recursos" (Naciones Unidas [NU], 2018, p. 51).

En este sentido, hay que aludir que, en acuerdo con Touguinha y Pato (2011), los escenarios actuales que se yerguen como la crisis socioambiental en que subsistimos, y que es patente en las ciudades, tiene su origen en el ser humano. Por esta razón, las expresiones escogidas por los participantes en el estudio se tornan en el comportamiento y preceptos a superar para el logro de contar con ciudades y comunidades sustentables. Es así que se halló un valor de J de 28 palabras distintas, que indican los desafíos que deberán superarse para alcanzar el Objetivo 
http://doi.org/10.15359/ree.25-3.37

http://www.una.ac.cr/educare

educare@una.ac.cr

11 de los ODS, desde los constructos derivados de los estudiantes universitarios participantes; de las cuales tuvieron una mayor frecuencia de menciones la contaminación del aire y basura, con $11.5 \%$ y $10.8 \%$ respectivamente. Integran el $22.3 \%$ de las alusiones realizadas por los encuestados, y que se instituyen como los retos principales de las ciudades y comunidades sustentables futuras.

Esta relevancia de la contaminación del aire, se sostiene en el marco de la realidad en que subsisten los estudiantes y donde se desarrolló el estudio, el cual es el Área Metropolitana de Monterrey (AMM), en el que la frecuencia de una mala calidad del aire es cada vez más habitual y, además, que en un estudio llevado a cabo recientemente "por la Organización Mundial de la Salud, en 1,600 ciudades ubicadas en 91 naciones, para determinar la calidad del aire, reporta particularmente que para México el AMM presenta la peor calidad" (Cantú-Martínez, 2015, p. 45). Esto se corroboró a través del Instituto Nacional de Estadística y Geografía (INEGl, 2017) que reportó para el AMM en 2016 una calidad no satisfactoria para las partículas con fracción respirable (PM10), partículas finas (PM2.5) y ozono, de 275, 69 y 59 días del año respectivamente, en las que se sobrepasó la norma y limitó las actividades al aire libre.

Por lo que respecta a la basura, también es consecuencia del contexto del AMM, donde se ha pronunciado por el Dr. Alfonso Martínez, director del Observatorio Ciudadano de la Calidad del Aire, que en Nuevo León una persona en promedio genera $1.8 \mathrm{~kg}$ diarios de residuos por día, y eso resulta indicador que está por encima de muchas ciudades en México (Walle, 2019). Para el AMM esto representa una problemática extremadamente fuerte, si consideramos que en 2015 el AMM contaba con 4245510 habitantes (INEGl, 2017) y al multiplicarse por el indicador estimado nos arroja una cantidad de generación de residuos de $7641918 \mathrm{~kg}$ diarios, de los cuales muchos de ellos son arrojados a la vías y áreas públicas, generando con ello inundaciones al recubrir los sistemas de alcantarillado.

Con lo anterior, se confirma lo que Rodríguez (2010) señala como la presencia de una memoria semántica. Esto señala los significados de las palabras y las vinculaciones de estas alusiones al marco de referencia socioambiental en el que se coexiste, puesto que, mediante estas, las personas conjugan sus experiencias, ideas y percepciones. $Y$, en este caso, los estudiantes universitarios construyen los retos de las ciudades y comunidades sustentables.

Por otra parte, todas las expresiones verbales adscritas al Grupo SAM que fueron las más sobresalientes, se vinculan a las metas de los ODS 11 denominado Ciudades y Comunidades Sustentables, que se ha suscrito en la Agenda 2030, con el propósito de evaluar los progresos en materia de sustentabilidad. Asimismo, se prueba, en relación al género, que el concepto detonante retos de las ciudades y comunidades sustentables, el cual se concibe en una orientación, tuvo influencia en las respuestas entre los estudiantes universitarios, con los cuales se demostró que pueden subsistir opiniones discrepantes, al solo coincidir en un $75 \%$, como lo indica Cantú-Martínez (2019; 2020) y Marroquín Ciendúa et al. (2019). 
http://doi.org/10.15359/ree.25-3.37

Por último, resulta importante hacer mención que las redes semánticas construyen el espacio subjetivo que se genera entre la vinculación que subsiste entre las actitudes y el comportamiento del ser humano, en el cual se muestra la forma de organizar el conocimiento, a la luz de una interiorización individual, y posteriormente colectiva, que le permite a toda persona llevar a cabo una composición y discernimiento del entorno que le circunda.

\section{Declaración de Material complementario}

Este artículo tiene disponible, como material complementario:

-La versión preprint del artículo en https://doi.org/10.5281/zenodo.4891003

\section{Referencias}

Aznar Minguet, P., Ull Solís, M.A., Martínez Agut, M. del P. y Piñero Guillamany, A. (2014). Competencias básicas para la sostenibilidad: Un análisis desde el diálogo disciplinar. Bordón, 66(2), 13-27. https://doi.org/10.13042/Bordon.2014.66201

Bonil, J.,Junyent, M. y Pujol, R. M. (2010). Educación para la sostenibilidad desdela perspectiva de la complejidad. Revista Eureka sobre Enseñanza y Divulgación de las Ciencias, 7(Extraordinario), 198-215. https://doi.org/10.25267/Rev Eureka ensen divulg cienc.2010.v7.iextra.05

Bottino Bernardi, R. (2009). La ciudad y la urbanización. Estudios Históricos, (2), 1-14. http://www. estudioshistoricos.org/edicion 2/rosario bottino.pdf

Cantú-Martínez, P. C. (2015). Escenario socioambiental del área metropolitana de Monterrey. Ciencia UANL, 18(72), 38-46. http://cienciauanl.uanl.mx/?p=3818

Cantú-Martínez, P. C. (2019). La apreciación semántica de la noción desafíos en bioética por alumnos universitarios del campo de las ciencias biológicas. Revista Iberoamericana de Bioética, (10), 01-11. https://doi.org/10.14422/rib.i10.y2019.002

Cantú-Martínez, P. C. (2020). Actitudes proambientales en jóvenes universitarios. Ciencia y Educación, 4(2), 67-74. https://doi.org/10.22206/cyed.2020.v4i2.pp67-74

Charry Joya, C. A. (2006). Perspectivas conceptuales sobre la ciudad y la vida urbana: El problema de la interpretación de la cultura en contextos urbanos. Antípoda, (2), 209-228. http:// www.scielo.org.co/pdf/antpo/n2/n2a12.pdf 
http://doi.org/10.15359/ree.25-3.37

http://www.una.ac.cr/educare

educare@una.ac.cr

Instituto Nacional de Estadística y Geografía. (2017). Anuario estadístico y geográfico de Nuevo León 2017. Autor. https://www.datatur.sectur.gob.mx/ITxEF Docs/NL ANUARIO PDF.pdf

Marroquín Ciendúa, F., Palacios Chavarro, J. A., Sandoval Escobar, M. C. y Sierra Puentes, M. C. (2019). Consumo ecológico: Estudio exploratorio sobre el comportamiento de mujeres en Colombia. Revista Espacios, 40(24), 1-14. http://www.revistaespacios.com/a19v40n24/ a19v40n24p24.pdf

Naciones Unidas. (2018). La agenda 2030 y los objetivos de desarrollo sostenible. Una oportunidad para América Latina y el Caribe. CEPAL. https://repositorio.cepal.org/bitstream/ handle/11362/40155/24/S1801141 es.pdf

Naciones Unidas. Asamblea General. (4 de agosto de 1987). A/42/427. Desarrollo y cooperación económica internacional: Medio ambiente. Informe de la Comisión Mundial sobre el medio ambiente y el desarrollo. https://tinyurl.com/y69vbgit

Programa de las Naciones Unidas para el Desarrollo. (2019a). Objetivos de desarrollo sostenible. https://www1.undp.org/content/undp/es/home/sustainable-development-goals.html

Programa de las Naciones Unidas para el Desarrollo. (2019b). Objetivo 11: Ciudades y comunidades sostenibles. https://www.undp.org/content/undp/es/home/sustainable-developmentgoals/goal-11-sustainable-cities-and-communities.html\#targets

Rodríguez, R. J. (2010). Herramientas informáticas para la representación del conocimiento. Subjetividad y Procesos Cognitivos, 14(2), 217-232. http://www.scielo.org.ar/scielo. php?script=sci arttext\&pid=S1852-73102010000200017

Touguinha, S. y Pato, C. (2011). Valores personales, creencias ambientales ecocéntricas y comportamiento ecológico de trabajadores brasileños: El caso del Ministerio Público del Distrito Federal y Territorios. Quaderns de Psicologia, 13(1), 35-45. https://www. quadernsdepsicologia.cat/article/view/918

Vera-Noriega, J. Á., Pimentel, C. E. y Batista de Albuquerque, F. J. (2005). Redes semánticas: Aspectos teóricos, técnicos, metodológicos y analíticos. Ra Ximhai, 1(3), 439-451. https:// doi.org/10.35197/rx.01.03.2005.01.jv

Walle, C. E. (27 de marzo de 2019). En NL una persona genera un kilogramo de basura.ABC Noticias. https://abcnoticias.mx/en-nl-una-persona-genera-un-kilogramo-de-basura/131535 